\title{
Crisis del coronavirus: impacto y medidas económicas en Europa y en el mundo
}

Crisis del coronavirus: impacto y medidas económicas en Europa y en el mundo Crise do coronavírus: impactos e medidas econômicas na Europa e nos mundo Coronavirus crisis: impacts and economic measures in Europe and in the world Crise de coronavirus: impacts et mesures économiques en Europe et dans le monde

\section{Javier Cifuentes-Faura}

\section{OpenEdition}

Journals

Edición electrónica

URL: http://journals.openedition.org/espacoeconomia/12874

DOI: 10.4000/espacoeconomia.12874

ISSN: 2317-7837

\section{Editor}

Núcleo de Pesquisa Espaço \& Economia

\section{Referencia electrónica}

Javier Cifuentes-Faura, « Crisis del coronavirus: impacto y medidas económicas en Europa y en el mundo », Espaço e Economia [Online], 18 | 2020, posto online no dia 20 abril 2020, consultado o 20 maio 2020. URL : http://journals.openedition.org/espacoeconomia/12874 ; DOI : https://doi.org/ 10.4000/espacoeconomia.12874

Este documento fue generado automáticamente el 20 mayo 2020.

(c) NUPEE 


\section{Crisis del coronavirus: impacto y medidas económicas en Europa y en el mundo}

Crisis del coronavirus: impacto y medidas económicas en Europa y en el mundo Crise do coronavírus: impactos e medidas econômicas na Europa e nos mundo Coronavirus crisis: impacts and economic measures in Europe and in the world Crise de coronavirus: impacts et mesures économiques en Europe et dans le monde

Javier Cifuentes-Faura

\section{INTRODUCCIÓN}

1 El covid-19 es un nuevo coronavirus que causa respiratorios, digestivos y sistemáticos que afectan la salud humana. Surgió en China y se ha expandido rápidamente a nivel mundial, afectando prácticamente a todos los países, y ocasionando millones de infectados y miles de personas fallecidas. La Organización Mundial de la Salud (OMS) declaró al problema como una emergencia sanitaria y la catalogó como pandemia a nivel internacional.

2 Se han puesto en marcha medidas de contención como el cierre de fábricas y oficinas, la prohibición de realizar viajes que no sean estrictamente necesarios, incluso se ha impuesto una cuarentena obligatoria en casa (Baldwin 2020, Baldwin y Weder di Mauro 2020), con el objetivo de reducir la pandemia.

3 Esta crisis sanitaria ha desembocado en una auténtica crisis económica sin precedentes. En el caso de la Unión Europea, se deben proporcionar medidas que garanticen cierta estabilidad financiera. En este trabajo repasamos algunas de las posibles medidas. Es importante recalcar que la moneda del euro se construyó sobre dos pilares que se complementan pero que a la vez son desiguales. Por un lado, una política monetaria única, en manos del BCE, y, por otro lado, políticas fiscales descentralizadas, a cargo de 
los Estados miembros de la Unión Europea, aunque limitadas por normas comunes. Esta arquitectura asimétrica tiene algunas deficiencias como la última crisis de la deuda soberana que creó incertidumbre sobre la permanencia de la moneda única. Para hacer frente a esto, se estableció en 2012 un prestamista fiscal de último recurso, el Mecanismo Europeo de Estabilidad (MEDE), que pretende proporcionar el apoyo necesario a los países de la UE con el objetivo de garantizar la estabilidad financiera de la zona del euro, y que ahora, podría ser de mucha utilidad en esta crisis.

La crisis del Coronavirus se prevé que sea la mayor crisis económica y el mayor desafío desde la Gran Recesión. Es una crisis sin precedentes por sus características. A la disminución de la capacidad productiva (shock de oferta), con un desplome de los mercados internacionales, y al declive de la demanda interna (shock de demanda), hay que añadirle las restricciones al movimiento de personas, que producen a la vez efectos sobre la oferta y la demanda.

\section{CRISIS ECONÓMICA DEL COVID-19}

5 Debido a la crisis sanitaria, la economía se ha visto drásticamente afectada. Los mercados financieros mundiales han registrado fuertes caídas, y la volatilidad se encuentra en niveles similares o incluso superiores a los de la crisis financiera que comenzó en 2008.

6 A diferencia del año 2008, en esta crisis el gran problema no se encuentra en los mercados financieros sino en la economía real, compuesta por las empresas, especialmente las pymes, y los trabajadores. Es una crisis sin precedentes, por lo que es difícil hacer comparaciones con otras crisis mundiales previas. Esta crisis económica, derivada de las consecuencias provocadas por la crisis sanitaria convertida en pandemia global, presenta unas características particulares, ya que no afecta solo a los países de ingresos bajos y medios. Se está produciendo simultáneamente la destrucción de la demanda y la oferta, y las tasas de interés están en mínimos históricos. En esta crisis, el mundo está mucho más integrado y están teniendo lugar efectos indirectos en las cadenas de suministro de todos los países.

7 Los mercados financieros se están desplomando, llevando a algunos a una crisis crediticia, particularmente en el sector del comercio minorista y el turismo. Están siendo también muy afectados los trabajadores autónomos. Muchos trabajadores están sufriendo también ajustes temporales de empleo, por lo que el trabajador se encuentra en situación de desempleo durante el tiempo que se haya considerado que la actividad estará frenada, es decir, mientras dure la declaración del estado de alarma como consecuencia del coronavirus.

8 El problema también se encuentra en que muchas empresas, de forma previa a la aparición del virus, dedicaron sus ingresos a recomprar sus propias acciones en lugar de invertirlo en innovación y en aspectos conducentes a realizar mejoras en su productividad, por lo que muchos mercados estaban infravalorando el riesgo de sus bonos y acciones.

9 América Latina es una de las zonas más vulnerables a esta crisis, cuyos niveles de pobreza se encuentran alrededor del $30 \%$ y la mayoría de la población está inmersa en el sector informal de la economía sin seguridad social o redes de protección. También son bastante vulnerables los países subdesarrollados o en vías de desarrollo. 
10 Los países latinoamericanos están tomando medidas económicas para proteger de las consecuencias derivadas del coronavirus. Aunque se necesitará una acción conjunta por parte de las instituciones financieras internacionales y de las calificadoras de riesgo, para evitar que continúe el aumento de la pobreza y la desigualdad.

11 La demanda se espera que afecte más a los bienes duraderos que a los no duraderos, especialmente al sector manufacturero. Las perturbaciones de la demanda se producirán por las caídas macroeconómicas de la demanda agregada debido a la recesión, y por retrasos en la compra por parte de los consumidores por motivos de precaución o de espera.

12 Las interrupciones directas de la oferta obstaculizan la producción ya que la enfermedad se sitúa en el centro manufacturero del mundo, China, aunque la situación del Covid-19 es cada vez mejor en el país asiático, pero se está extendiendo rápidamente a otros gigantes industriales como Estados Unidos. Este contagio en la cadena de suministro producirá choques de suministro directo entre los países menos afectados por la pandemia, que encontrarán más difícil y más caro adquirir los materiales industriales necesarios para la fabricación, y los países más afectados, que venderán estos insumos a un precio más caro por la limitación y escasez de unidades.

13 Las exportaciones bilaterales se reducirán drásticamente debido a perturbaciones de la oferta, es decir, cambios en el PIB en el país de origen - el vendedor - y choques de demanda, cambios en el PIB en el país de destino - el comprador -. La economía de los países se podrá ver afectada a la vez por un shock de oferta y de demanda. Si el COVID-19 produce un shock de oferta, las exportaciones caerán, y se reducirán especialmente en aquellos países más afectados. Si la crisis del COVID-19 se ve traducida en un choque de demanda, las importaciones serán las que sufran esta caída, y se reducirán la mayoría de los socios comerciales. Las naciones más afectadas provocarán grandes caídas en los flujos comerciales a nivel mundial. En el gráfico 1 se puede observar la relación entre el crecimiento del comercio mundial de mercancías y el crecimiento del PIB mundial en los últimos años, donde se observa una gran caída en 2008, y en la actualidad. 
Gráfico 1. Relación entre el crecimiento del comercio mundial de mercancías y el crecimiento del PIB mundial (1990-2020)

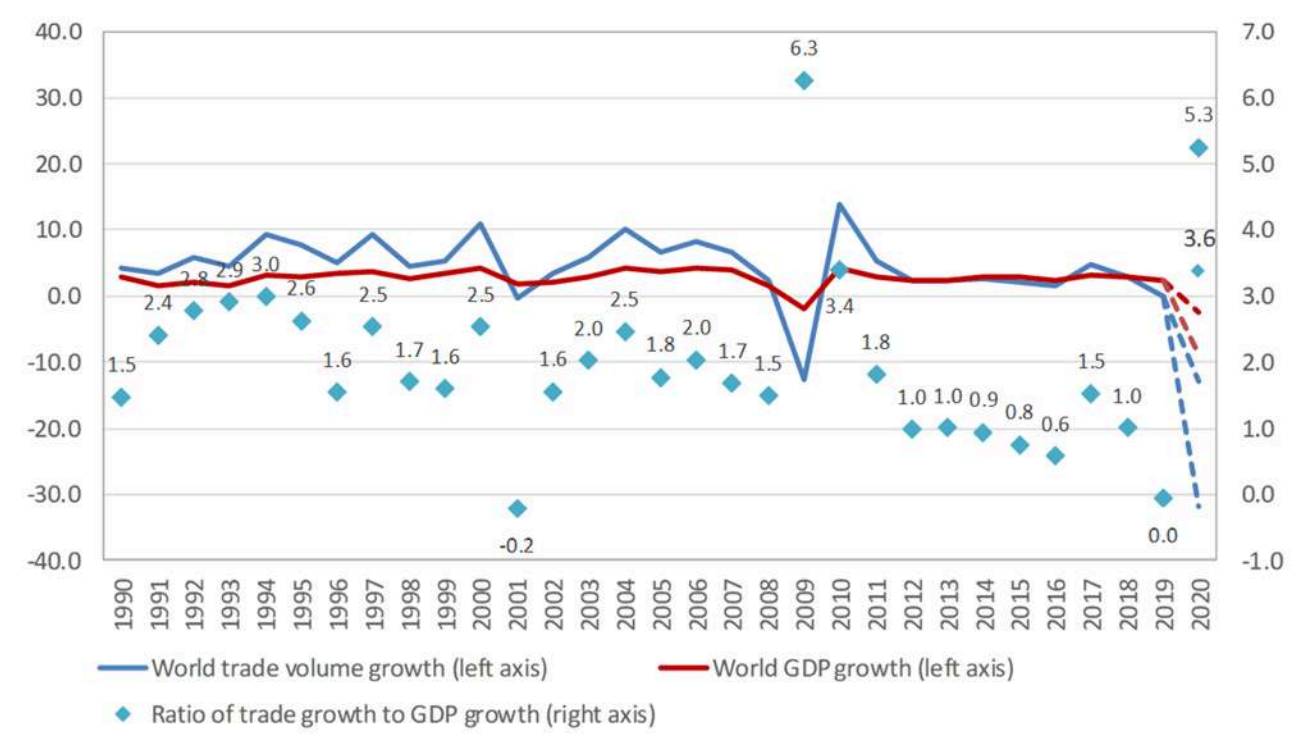

Fuente: Secretaría de Comercio de la OMC y estimaciones de consenso para el PIB histórico. Proyecciones del PIB basadas en escenarios simulados con el modelo de comercio mundial de la OMC.

Esta recesión económica también viene marcada por el papel de las cadenas de valor y el comercio de servicios. Cuando el coronavirus se limitaba solo a China ya existía una interrupción de la cadena de valor. Con la generalización del virus, el comercio se reducirá aún más en aquellos sectores caracterizados por vínculos complejos en la cadena de valor, especialmente los productos electrónicos y automovilísticos. Es probable que las importaciones de los principales insumos de producción se vean interrumpidas por la paralización económica y el distanciamiento social, que ha provocado el cierre temporal de fábricas y empresas en China, y que ahora está ocurriendo en Europa y América del Norte. Tanto las empresas que operan a nivel mundial como las locales deben afrontar el reto de gestionar esta interrupción de la cadena de suministro, realizando cálculos de la eficiencia económica en función del riesgo por parte de cada empresa.

Las expectativas futuras son toda una incógnita. La crisis del CoVID-19 tiene a los consumidores y las empresas en una incertidumbre continua. Algunos indicadores, como el Índice de Gerentes de Compras (Purchasing Manager Index -PMI-), han bajado drásticamente. Los datos mensuales que se publican del PMI proceden de encuestas a altos ejecutivos de empresas del sector privado. A partir del PMI se puede determinar si una economía goza de una buena salud, y tomar medidas de forma previa si los datos indican una posible recesión, por eso este indicador es especialmente importante para poder aventurarse a una crisis económica y reducir su impacto impulsando medidas. Los datos del PMI son utilizados por los profesionales financieros para comprender cuál es el rumbo y las expectativas de cada economía.

Las repercusiones del brote de COVID-19 en el comercio internacional todavía no son visibles en la mayoría de los datos comerciales, pero a partir de algunos indicadores como el PMI se puede conocer el alcance de la desaceleración mundial que se está produciendo. El PMI mundial de JP Morgan de marzo mostró que las órdenes de exportación en el sector manufacturero se hundían a 43,3 en relación con un valor de 
referencia de 50, y que los nuevos negocios de exportación de servicios caían a 35,5, lo que sugiere una grave desaceleración (Gráfico 2).

Gráfico 2: Nuevos pedidos de exportación de los Índices de Gerentes de Compras (PMI) 2008-2020.

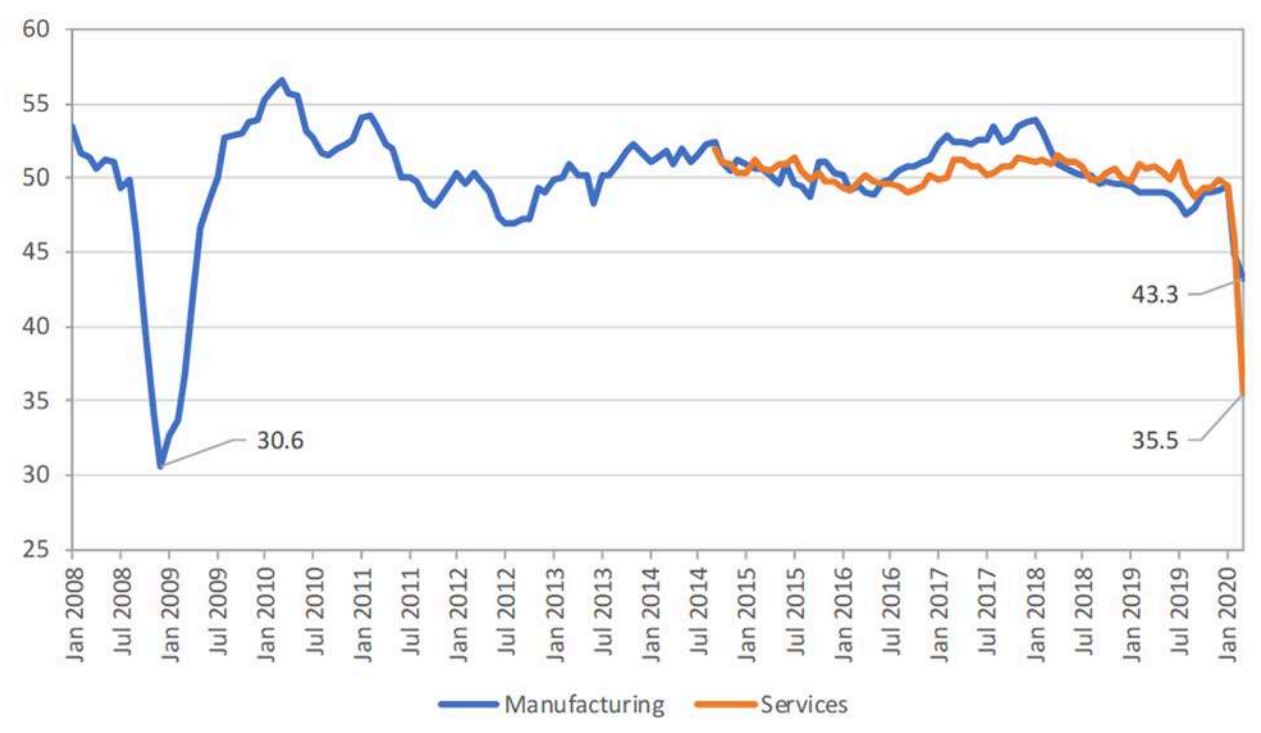

Fuente: IHS Markit

\section{CORONAVIRUS EN EUROPA}

Se estima que en Europa las previsiones de crecimiento del PIB serán negativas para el conjunto de la eurozona del $-4 \%$ en 2020 y una recuperación positiva con un crecimiento del $2 \%$ en 2021. Estas hipótesis son menos pesimistas que las que manejan entidades reputadas como Morgan Stanley (-5\% para 2020), Capital Economics (-6\%) o JP Morgan (-11,4\%).

Sin embargo, debido a la situación actual del coronavirus, el MEDE podría intervenir debido la presión existente. De eta forma, estos países podrán tener acceso a 500.000 millones de euros en las mejores condiciones de financiación posibles, lo cual se haría cumpliendo con lo terminado en el Tribunal de Justicia de las Comunidades Europeas, y seguirían estando obligados a reembolsar los préstamos.

La respuesta a la crisis derivada del coronavirus sigue siendo una incógnita por parte de Europa. Los préstamos por parte del Mecanismo Europeo de Estabilidad es una de las opciones que se contemplan. Otra opción factible es la de los bonos de coronavirus, conocidos como "coronabonos" (Bénassy-Quéré 2020, Bofinger et al. 2020, Erce et al. 2020, Grund et al. 2020). Esta emisión única de bonos europeos mutuos podría crear un peligroso precedente de mutualización de la deuda.

Desde la década de los 70, la Comisión Europea ha colocado en los mercados privados varios bonos comunitarios, los cuales han estado garantizados por los Estados miembros y se han distribuido en aquellos países que se encontraban en crisis. Estos bonos fueron reembolsados por completo en el pasado. Los bonos comunitarios suponen una responsabilidad conjunta y solidaria por parte de todos los estados, y es una forma de cooperar financieramente de modo solidario en Europa. 
21 Aunque supondría cierta coordinación y solidaridad entre los Estados miembros, la línea de crédito específica reduciría los riesgos para la estabilidad económica y financiera de todos, al tiempo que permitiría a los miembros mantener sus esfuerzos haciendo que el coste de endeudarse dependiera menos de las situaciones fiscales individuales.

\section{CONCLUSIONES}

22 La crisis del Covid-19 es una crisis sin precedentes, por lo que es difícil hacer comparaciones con otras crisis mundiales previas. La economía real, compuesta por empresas y trabajadores, está siendo la más afectada. Esta crisis está afectando a todos los países, tanto a las principales potencias económicas como a los países en vías de desarrollo. Se está produciendo al mismo tiempo una destrucción de la demanda y de la oferta, y los mercados financieros se están desplomando, llevando a algunos a una crisis crediticia, particularmente en el sector del comercio minorista y el turismo.

El sector servicios será el más afectado en todos los países afectados, especialmente debido a la imposición de restricciones al transporte y los viajes y el cierre de muchos establecimientos minoristas y de hostelería. El comercio de mercancías será imposible de mantener si no se prestan servicios como el transporte. A diferencia de las mercancías, no hay inventarios de servicios que se puedan retirar hoy y reponer en una etapa posterior, pudiendo ser irrecuperable esta disminución del comercio de servicios durante esta crisis sanitaria y económica. Sin embargo, existen algunos servicios que podrían beneficiarse de esta crisis, como es el caso de los servicios derivados de las tecnologías de la información y la comunicación, cuya demanda ha aumentado de forma considerable, debido por un lado a la necesidad de las personas de entretenerse, socializar y comunicarse a distancia, y, por otro lado, por el fomento del teletrabajo que permite que los empleados trabajen desde sus casas sin tener que acudir de forma presencial al centro de trabajo.

24 Será necesario cierta coordinación y solidaridad entre todos los Estados y proponer medidas económicas que reduzcan el impacto de la crisis económica que se avecina. En el caso de Europa, los préstamos por parte del Mecanismo Europeo de Estabilidad es una de las opciones más viables.

\section{BIBLIOGRAFÍA}

Baldwin, R (2020). It's not exponential: an economist's view of the epidemiological curve. VoxEU.org, 12 de marzo.

Baldwin, R y B Weder di Mauro (2020). Economics in the Time of COVID-19, CEPR Press.

Bénassy-Quéré, A, A Boot, A Fatás, M Fratzscher, C Fuest, F Giavazzi, R Marimon, P Martin, J Pisani-Ferry, L Reichlin, D Schoenmaker, P Teles and B. Weder di Mauro (2020), "COVID-19: A proposal for a Covid Credit Line", VoxEU.org, 21 March. 
Bofinger, P, S Dulline, G Felbermayr, M Huther, M Schularick, J Sudekum and C Trebesh (2020), “ To avoid economic disaster, Europe must demonstrate financial solidarity”, NewStatesman, 21 March.

Capital Economics (2020). EZ facing more than 10\% quarterly slump in GDP, 17 de marzo. https:// www. capitaleconomics.com/publications/europeaneconomics/european-economics-update/ezfacingmore-than-10-quarterly-slump-in-gdp/

Erce, A, A Garcia Pascual and R Marimon (2020), "The ESM can finance the COVID fight now", VoxEU.org, 06 April.

Grund, S, L Guttenberg and C Odendahl (2020), "Sharing the fiscal burden of the crisis: A Pandemic Solidarity Instrument for the EU”, VoxEU.org, 05 April.

JP Morgan (2020). Assessing the fallout from the Coronavirus pandemic, 20 de marzo. Disponible en: https://www. jpmorgan.com/global/research/coronavirus-impact

Markets Insider (2020). Morgan Stanley officially says a global recession is now its base case as the coronavirus outbreak escalates, 17 de marzo. https://markets. businessinsider.com/news/ stocks/coronavirus-fuelrecession-economic-forecast-us-europe-china-

morganstanley-2020-3-1029004569

\section{RESÚMENES}

La crisis del Coronavirus se prevé que sea la mayor crisis económica y el mayor desafío desde la Gran Recesión. Es una crisis sin precedentes por sus características. A la disminución de la capacidad productiva, con un desplome de los mercados internacionales, y al declive de la demanda interna, hay que añadirle las restricciones al movimiento de personas, que están produciendo al mismo tiempo efectos sobre la oferta y la demanda.

Es necesario que se tomen medidas económicas para disminuir los efectos que va a producir esta pandemia a nivel mundial, aunque la respuesta por parte de algunos países es una incógnita. En el caso de Europa, los préstamos por parte del Mecanismo Europeo de Estabilidad son una de las opciones que se contemplan. Otra opción factible es la de los coronabonos.

Espera-se que a crise do Coronavírus seja a maior crise econômica e o maior desafio desde a Grande Recessão. É uma crise sem precedentes por suas características. Para além do declínio da capacidade produtiva, com o colapso dos mercados internacionais, e do declínio da demanda interna, as restrições à circulação de pessoas estão produzindo ao mesmo tempo efeitos negativos tanto na oferta como na procura.

É necessário tomar medidas econômicas para atenuar os efeitos que esta pandemia terá no mundo, ainda que a resposta de alguns países seja uma incógnita. No caso da Europa, a concessão de empréstimos pelo Mecanismo de Estabilidade Europeu é uma das opções a considerar. Outra opção viável é a dos coronabônus.

The Coronavirus crisis is expected to be the biggest economic crisis and the biggest challenge since the Great Recession. It is an unprecedented crisis in terms of its characteristics. In addition to the decline in productive capacity, with a collapse of international markets, and the decline in domestic demand, restrictions on the movement of people are having an impact on both supply and demand.

Economic measures need to be taken to lessen the effects that this pandemic will have on the world, although the response by some countries is unknown. In the case of Europe, lending by 
the European Stability Mechanism is one of the options being considered. Another feasible option is that of coronabonds.

La crise du coronavirus devrait être la plus grande crise économique et le plus grand défi depuis la Grande Récession. Il s'agit d'une crise sans précédent de par ses caractéristiques. Outre le déclin de la capacité de production, avec l'effondrement des marchés internationaux, et la baisse de la demande intérieure, les restrictions à la circulation des personnes ont un impact à la fois sur l'offre et sur la demande.

Des mesures économiques doivent être prises pour atténuer les effets que cette pandémie aura sur le monde, bien que la réaction de certains pays soit inconnue. Dans le cas de l'Europe, l'octroi de prêts par le mécanisme de stabilité européen est l'une des options envisagées. Une autre option possible est celle des coronabonds.

\section{ÍNDICE}

Mots-clés: Covid-19, crise économique, mesures économiques, Europe.

Palabras claves: Covid-19, crisis económica, medidas económicas, Europa.

Keywords: Covid-19, economic crisis, economic measures, Europe.

Palavras-chave: Covid-19, crise económica, medidas económicas, Europa.

\section{AUTOR}

\section{JAVIER CIFUENTES-FAURA}

Universidad de Murcia 